\title{
Early Single-Center Experience with Robotic Partial Nephrectomy Using the da Vinci Xi: Comparative Assessment with Conventional Open Partial Nephrectomy
}

\author{
Daisuke Motoyama Ryota Aki Yuto Matsushita Keita Tamura \\ Toshiki Ito Takayuki Sugiyama Atsushi Otsuka Hideaki Miyake
}

Department of Urology, Hamamatsu University School of Medicine, Hamamatsu, Japan

\section{Key Words}

Robotic partial nephrectomy • Open partial nephrectomy • da Vinci Xi • Trifecta • Propensity score-matched analysis

\begin{abstract}
Background: The objective of this study was to evaluate our experience with robot-assisted partial nephrectomy (RAPN) in comparison with conventional open partial nephrectomy (OPN). Patients and Methods: This study included 37 and 50 patients undergoing OPN and RAPN for small renal masses, respectively. A single surgeon performed RAPN for all 50 cases using the da Vinci Xi. Trifecta was defined as satisfying all of the following 3 criteria: ischemic time of $\leq 25 \mathrm{~min}$ utes, negative surgical margin and no major postoperative complications. Results: After adjusting patient variables by 1:1 propensity-score matching, 37 patients were included in each group, and no significant differences in major clinicopathological characteristics were noted between these 2 groups. RAPN was significantly superior to OPN with respect to operative time, estimated blood loss and postoperative length of hospital stay. The rate of trifecta achievement was significantly higher in the RAPN group than in the OPN group (91.9 vs. $62.2 \%)$. Furthermore, the operative procedure and R.E.N.A.L. nephrometry score were found to be independently associated with trifecta outcome by multivariate analysis of the entire cohort. Conclusions: Although
\end{abstract}

\section{KARGER}

Fax +4161306 1234

E-Mail karger@karger.com

www.karger.com
(C) 2019 The Author(s)

Open access

This article is licensed under the Creative Commons AttributionNonCommercial-NoDerivatives 4.0 International License (CC BYNC-ND) (http://www.karger.com/Services/OpenAccessLicense). Usage and distribution for commercial purposes as well as any distribution of modified material requires written permission. this is our early experience with 50 initial cases, RAPN using the da Vinci Xi resulted in more favorable perioperative outcomes than OPN.

(c) 2019 The Author(s)

Published by S. Karger AG, Basel

\section{Introduction}

Partial nephrectomy (PN) is currently recognized as the standard of care for treating T1a renal tumors and an alternative option for T1b tumors, as PN has been reported to produce equivalent oncological outcomes with the additional benefit of preserving renal function compared with radical nephrectomy [1]. Furthermore, during the last 2 decades, minimally invasive approaches, particularly robot-assisted PN (RAPN), have gained wide acceptance as promising surgical options for patients with small renal tumors due to advancements in surgical techniques and instruments $[2,3]$. Indeed, there have been several studies in which the clinical outcomes of RAPN were superior to those of other surgical procedures [3-6]. For example, Wu et al. [4] conducted a systematic review and meta-analysis, and reported more favorable findings for RAPN, including fewer perioperative complications, less intraoperative estimated blood loss and shorter postoperative hospitalization period, than those for conventional open partial nephrectomy.

Daisuke Motoyama, MD

Department of Urology, Hamamatsu University School of Medicine 
Since its introduction for several surgical procedures, the da Vinci robotic system has undergone a number of refinements each with significant technological advancement over previous versions [7]. The da Vinci $\mathrm{Xi}$ is the latest robotic platform characterized by several advantages, such as thinner mounted robotic arms with additional joints on a movable overhead boom, which enables surgeons to perform more precise surgical procedures by markedly reducing technical difficulty [8]. Such features are likely to be beneficial for RAPN, which requires highly complicated surgical procedures using multiple robotic arms in a comparatively close space [9]. However, to date, information on the clinical outcomes of RAPN in clinical practice using the da Vinci $\mathrm{Xi}$ is limited.

Considering previous reports, we herein describe our initial experience with RAPN using the da Vinci Xi surgical system for patients with small renal masses, and compared perioperative outcomes between RAPN and conventional OPN performed at our institution.

\section{Patients and Methods}

The design of this study was approved by the Research Ethics Committee of our institution (Approval Number: E15-115), and the need to obtain informed consent for involvement in this study from all of the included patients was waived due to its retrospective design. This study included a total of 87 consecutive patients with small renal masses, consisting of 37 and 50 who underwent conventional OPN and RAPN, respectively, at our institution between July 2010 and August 2017.

All patients underwent preoperative imaging examinations with contrast-enhanced computed tomography (CT) and/or magnetic resonance imaging. All data on these 87 patients regarding clinical characteristics, as well as operative and postoperative findings were collected from their medical records. For each patient, the Chronic Kidney Disease Epidemiology formula [10] was used to calculate the estimated glomerular filtration rate, and the R.E.N.A.L. nephrometry score was assessed according to the preoperative tumor characteristics on imaging examinations, as previously described [11]. Postoperative complications were evaluated based on the Clavien-Dindo system [12]. In this series, the outcomes of the trifecta, a widely accepted key surrogate for successful PN, were defined as satisfaction of the 3 following factors: ischemic time $\leq 25$ minutes, negative surgical margins and no major postoperative surgical complications corresponding to Clavien-Dindo system $\geq 3$. To minimize selection bias between the OPN and RAPN groups, the following patient characteristics were adjusted using 1:1 propensity-score matching: sex, age, body mass index, tumor side, hilar tumor, solitary tumor and R.E.N.A.L. nephrometry score.

In general, OPN was performed using the flank retroperitoneal approach according to a previously reported procedure with a minor modification [13]. Briefly, ice slush was placed around the kidney, and under cold ischemic conditions by clamping the renal artery, the tumor was excised with an adequate surrounding mar- gin of normal renal tissue. Opened calyces and bleeding sites were sutured, and the parenchymal defect was closed with horizontal interrupted sutures.

RAPN was performed by a single surgeon (H.M.) employing a 3-arm da Vinci Xi robot system (Intuitive Surgical Inc., Sunnyvale, California, USA) under a pneumoperitoneum of $12 \mathrm{mmHg}$. Prior to RAPN, 3-dimensional (3D) images were reconstructed based on DICOM data of contrast-enhanced CT using the SYNAPSE VINCENT base system (Fujifilm, Inc., Tokyo, Japan), and were directly visualized on the screen of a surgeon's console with TilePro multi-input display functions. In this series, a transperitoneal approach was likely to be selected, except for patients with dorsal hilar tumors. In addition, 1 to 3 additional trocars, including AirSeal iFS (CONMED Japan KK, Tokyo, Japan), were placed for use by assistants. Briefly, to evaluate the tumor depth and plan the excision margins, an ultrasound probe, ARIETTA 70 (Hitachi, Inc., Tokyo, Japan), was used. Under complete clamping of the renal artery with the Bulldog, the tumor was excised with cold scissors with a secure margin of approximately $5 \mathrm{~mm}$. The collecting system and large vessels were repaired by 3-0 V-Loc sutures (COVIDIEN Japan, Inc., Tokyo, Japan), and renorraphy was then performed with 2-0 V-Loc for cross-compression along the defect.

All statistical analyses were performed using R ver. 3.1.1 software (R Development Core Team, https://www.r-project.org/index.html), and a p-value $<0.05$ was considered significant. Differences between the 2 groups were compared using the chi-square test or Mann-Whitney U test. Logistic regression analyses were used to calculate the propensity-score and to analyze the significance of predictors for trifecta achievement.

\section{Results}

Table 1 summarizes the major clinical characteristics of patients included in this study. In the entire cohort consisting of 37 and 50 patients undergoing OPN and RAPN, respectively, the proportion of patients with solitary kidney in the OPN group was significantly higher than that in the RAPN group. After 1:1 propensity-score matching, 37 patients were assigned to each group, and there were no significant differences in any parameter, including the proportion of solitary kidney, between the OPN and RAPN groups.

Table 2 presents the comparison of surgical and pathological outcomes between the OPN and RAPN groups after matching. A significant difference in the surgical approach was noted between the OPN and RAPN groups; the majority of patients in the OPN and RAPN groups underwent surgery by the retroperitoneal and transperitoneal approaches, respectively. RAPN was significantly superior to OPN with respect to operative time, estimated blood loss and postoperative length of hospital stay; however, there were no significant differences in the remaining outcomes between the 2 groups. 
Table 1. Patient and tumor characteristics

\begin{tabular}{|c|c|c|c|c|c|c|}
\hline & \multicolumn{3}{|c|}{ Entire cohort } & \multicolumn{3}{|c|}{ Propensity-score matched cohort } \\
\hline & $\begin{array}{l}\text { OPN } \\
(\mathrm{n}=37)\end{array}$ & $\begin{array}{l}\text { RAPN } \\
(\mathrm{n}=50)\end{array}$ & $\mathrm{p}$ & $\begin{array}{l}\text { OPN } \\
(\mathrm{n}=37)\end{array}$ & $\begin{array}{l}\text { RAPN } \\
(\mathrm{n}=37)\end{array}$ & $\mathrm{p}$ \\
\hline Sex, n (\%) & & & 0.44 & & & 1.00 \\
\hline Male & $23(62.2)$ & $35(70.0)$ & & $23(62.2)$ & $22(59.5)$ & \\
\hline Female & $14(37.8)$ & $15(30.0)$ & & $14(37.8)$ & $15(40.5)$ & \\
\hline Median age, year (range) & $64(33-78)$ & $65(36-90)$ & 0.27 & $64(33-78)$ & $61(36-90)$ & 0.92 \\
\hline Median BMI, $\mathrm{kg} / \mathrm{m}^{2}$ (range) & $23.3(16.5-29.9)$ & $23.7(16.0-33.5)$ & 0.53 & $23.3(16.5-29.9)$ & $23.4(16.0-33.0)$ & 0.91 \\
\hline Tumor side, $\mathrm{n}(\%)$ & & & 0.62 & & & 0.47 \\
\hline Right & $21(56.8)$ & $31(62.0)$ & & $21(56.8)$ & 25 (67.6) & \\
\hline Left & $16(43.2)$ & $19(38.0)$ & & $16(43.2)$ & $12(32.4)$ & \\
\hline Hilar tumor, $\mathrm{n}(\%)$ & $8(21.6)$ & $4(8.0)$ & 0.11 & 8 (21.6) & $4(10.8)$ & 0.35 \\
\hline Solitary kidney, n (\%) & $5(13.5)$ & $1(2.0)$ & 0.036 & $5(13.5)$ & $1(2.7)$ & 0.20 \\
\hline Median tumor size, mm (range) & $24(9-73)$ & $22(9-45)$ & 0.42 & $24(9-73)$ & $22(9-45)$ & 0.77 \\
\hline $\mathrm{T}$ classification, $\mathrm{n}(\%)$ & & & 0.11 & & & 0.29 \\
\hline cT1a & $29(78.4)$ & $46(92.0)$ & & $29(78.4)$ & $33(89.2)$ & \\
\hline cT1b & $6(16.2)$ & $4(8.0)$ & & $6(16.2)$ & $4(10.8)$ & \\
\hline cT2a & $2(5.4)$ & $0(0.0)$ & & $2(5.4)$ & $0(0.0)$ & \\
\hline Median R.E.N.A.L, score (range) & $7(4-10)$ & $7(4-10)$ & 0.41 & $7(4-10)$ & $7(4-10)$ & 0.87 \\
\hline
\end{tabular}

$\mathrm{BMI}=$ Body mass index .

Table 2. Surgical and pathological outcomes of propensity-score matched patients

\begin{tabular}{|c|c|c|c|}
\hline & $\begin{array}{l}\text { OPN } \\
(\mathrm{n}=37)\end{array}$ & $\begin{array}{l}\text { RAPN } \\
(\mathrm{n}=37)\end{array}$ & $\mathrm{p}$ \\
\hline Surgical approach, n (\%) & & & $<0.001$ \\
\hline Transperitoneal & $4(10.8)$ & $29(78.4)$ & \\
\hline Median operative time, $\min$ (range) & $210(129-361)$ & $176(118-275)$ & 0.032 \\
\hline Median console time, min (range) & - & $112(58-190)$ & - \\
\hline Median estimated blood loss, $\mathrm{ml}$ (range) & $360(35-3,476)$ & $35(0-500)$ & $<0.001$ \\
\hline Histological subtype, n (\%) & & & 0.85 \\
\hline Clear cell RCC & $28(75.7)$ & $25(67.6)$ & \\
\hline Other RCC & $3(8.1)$ & $5(13.5)$ & \\
\hline Benign tumor & $6(16.2)$ & $7(18.9)$ & \\
\hline Positive surgical margins, $\mathrm{n}(\%)$ & $3(8.1)$ & $0(0.0)$ & 0.24 \\
\hline Intraoperative blood transfusion, $\mathrm{n}(\%)$ & $4(10.8)$ & $0(0.0)$ & 0.12 \\
\hline Conversion to nephrectomy, n (\%) & $1(2.7)$ & $0(0.0)$ & 1.00 \\
\hline Achievement of trifecta outcomes, $\mathrm{n}(\%)$ & $23(62.2)$ & $34(91.9)$ & 0.0057 \\
\hline
\end{tabular}

$\mathrm{RCC}=$ Renal cell carcinoma; eGFR = estimated glomerular filtration rate. 
Table 3. Predictive factors for achieving trifecta outcomes in all patients

\begin{tabular}{|c|c|c|c|c|c|c|}
\hline & \multicolumn{3}{|c|}{ Univariate analysis } & \multicolumn{3}{|c|}{ Multivariate analysis } \\
\hline & Odds Ratio & $95 \% \mathrm{CI}$ & $\mathrm{p}$ & Odds Ratio & $95 \% \mathrm{CI}$ & $\mathrm{p}$ \\
\hline Age (> 65 vs. $<65$ years) & 1.43 & $(0.45-4.85)$ & 0.60 & - & - & - \\
\hline Sex (male vs. female) & 0.46 & $(0.10-1.67)$ & 0.27 & - & - & - \\
\hline BMI $\left(>22.0\right.$ vs. $\left.<22.0 \mathrm{~kg} / \mathrm{m}^{2}\right)$ & 1.61 & $(0.46-5.31)$ & 0.40 & - & - & - \\
\hline Hypertension (positive vs. negative) & 1.35 & $(0.43-4.58)$ & 0.61 & - & - & - \\
\hline Diabetes (positive vs. negative) Preoperative & 0.80 & $(0.22-3.28)$ & 0.76 & - & - & - \\
\hline CKD (positive vs. negative) & 1.92 & $(0.52-8.98)$ & 0.40 & - & - & - \\
\hline Solitary kidney (positive vs. negative) & 0.25 & $(0.03-2.05)$ & 0.12 & - & - & - \\
\hline Tumor side (right vs. left) & 2.49 & $(0.79-8.22)$ & 0.11 & - & - & - \\
\hline Hilar tumor (positive vs. negative) & 0.82 & $(0.18-5.22)$ & 0.72 & - & - & - \\
\hline R.E.N.A.L score (4-6 vs. 7-12) & 5.51 & $(1.16-52.98)$ & 0.026 & 5.27 & $(1.08-25.80)$ & 0.040 \\
\hline Operative procedure (RAPN vs. OPN) & 5.58 & $(1.56-25.61)$ & 0.0032 & 5.25 & $(1.63-16.90)$ & 0.0055 \\
\hline
\end{tabular}

$\mathrm{CI}=$ Confidence interval; $\mathrm{BMI}=$ body mass index $; \mathrm{CKD}=$ chronic kidney disease; $\mathrm{RAPN}=$ robot-assisted partial nephrectomy; OPN = open partial nephrectomy.

As major postoperative complications corresponding to Clavien-Dindo classification $\geq 3$, following 2 cases were experienced: methicillin-resistant staphylococcus aureus infected retroperitoneal abscess following urine leakage in OPN and hemorrhagic shock following renal artery pseudoaneurysm in RAPN, requiring 58 and 25 days of hospital stay after surgery, respectively. Furthermore, 23 $(62.2 \%)$ and $34(91.9 \%)$ patients in the OPN and RAPN groups, respectively, were judged to have achieved the trifecta, and the difference in the trifecta outcomes between the 2 groups was significant.

Uni- and multi-variate logistic regression analyses were performed to identify predictive factors of trifecta outcome in the entire cohort (table 3). Of several factors examined, univariate analyses identified the R.E.N.A.L. nephrometry score (4-6 vs. $\geq 7$ ) and surgical procedure (OPN vs. RAPN) as significant predictors of trifecta outcome, both of which were demonstrated to be independently associated with the achievement of the trifecta on multivariate analysis.

\section{Discussion}

Accumulating evidence has clarified the advantages of PN, which enables provision of equivalent oncological and superior renal functional outcomes compared with those of radical nephrectomy; thus, PN is currently recognized as the standard of care for patients with small localized renal tumors [1]. In recent years, the proportion of patients with small renal masses receiving PN by several minimally invasive procedures, particularly RAPN, is markedly increasing $[2,3]$. Indeed, the da Vinci robotic surgical systems, including the older systems $\mathrm{S}$ and $\mathrm{Si}$, have offered a magnified $3 \mathrm{D}$ view of the operative field with an extremely flexible wristed-instrument motion $[10,11]$, and there have been a number of studies documenting the usefulness of RAPN compared with other surgical approaches for PN [3-6]. Furthermore, further improvement in clinical outcomes of RAPN is expected with the introduction of the latest robotic system, da Vinci $\mathrm{Xi}$, which facilitates proper performance of the complex RAPN procedures with a shorter learning curve due to several innovations such as thinner mounted robotic arms with additional joints on a movable overhead boom [11]. Taken together, we retrospectively assessed our initial experience with RAPN for small renal masses using the da Vinci Xi system, and evaluated the significance of RAPN by comparing the clinical outcomes of patients undergoing conventional OPN and RAPN.

At our institution, PN for small renal masses was performed by conventional OPN prior to the initiation of RAPN, and RAPN was started in April 2016 after the introduction of the da Vinci Xi system, and the initial 50 patients who underwent RAPN were included in this study. When directly comparing characteristics between the OPN and RAPN groups, they were not completely consistent with each other; therefore, propensity-score matching was performed to select cohorts with common backgrounds. In this series, the superiority of RAPN to 
OPN in several important aspects, including operative time, estimated blood loss and postoperative length of hospital stay, was demonstrated after matching. Moreover, despite the lack of significant differences, the superiority of RAPN was also suggested by most of the remaining outcomes examined in this study, such as ischemic time, positive surgical margin and intraoperative blood transfusion. Collectively, these findings indicate that RAPN for patients with small renal masses achieves equivalent or superior perioperative outcomes compared with OPN; accordingly, RAPN may be recommended as the preferred surgical approach for these patients.

Currently, the achievement of the trifecta is accepted as an optimal indicator of short-term surgical outcome in patients undergoing PN [12]. In this series, the rates of trifecta achievement in the OPN and RAPN groups after matching were 62.2 and $91.9 \%$, respectively, and this difference was significant. To date, there have been several studies evaluating the trifecta achievement rates after RAPN, ranging between 43.3 and 82.6\% [13-19]. Such a wide range in the distribution of these rates can be explained by several differences among previous studies [13-19] such as experience of involved surgeons, surgical technique and characteristics of tumors. Considering the initial 50 cases assessed in this series, our trifecta outcome after RAPN is relatively favorable. Although it is difficult to clearly explain this finding, the following factors may be involved in the achievement of trifecta outcome: 1) an experienced surgeon who performed $>300$ robot-assisted radical prostatectomies performed RAPN for all 50 patients included in this study; 2) only the da Vinci Xi was used for RAPN for all 50 patients; 3) the proportion of patients with technically-difficult complex tumors was relatively low.

It is important to identify factors associated with trifecta outcomes in patients undergoing PN. In this series, the R.E.N.A.L. nephrometry score (4-6 vs. $\geq 7$ ) and surgical procedure (OPN vs. RAPN) were identified as independent predictors of trifecta outcomes after PN. To date, there have been limited studies analyzing predictors of trifecta outcomes after RAPN [16, 17, 20]. For example, Carneiro et al. [20] reported similar findings with study, i.e., robot use has a significant effect on achieving the trifecta based on multivariate analysis of data from patients undergoing either RAPN or laparoscopic PN. However, it is necessary to perform a prospective study with a much larger number of patients undergoing RAPN to draw a definitive conclusion on this point. Furthermore, it is also highly recommended to investigate over $90 \%$ preservation of the estimated glomerular filtration

Robotic Partial Nephrectomy Using the da Vinci Xi rate and no stage upgrade of chronic kidney disease at 1 year after surgery in addition to the trifecta as pentafecta outcomes to more precisely evaluate the significance of RAPN for small renal masses.

Another point of interest is to analyze the impact of the da Vinci Xi surgical system on perioperative outcomes of patients undergoing RAPN. As the da Vinci $\mathrm{Xi}$ surgical system was used for all patients undergoing RAPN in this series, it is impossible to compare the utility of the da Vinci Xi with that of the previous versions $\mathrm{S}$ or $\mathrm{Si}$. However, we consider the technical difficulty of RAPN to be significantly reduced by using the $\mathrm{Xi}$ system. In particular, the use of the $\mathrm{Xi}$ system enables the instruments to smoothly work without colliding with each other in a narrow operative field during RAPN, irrespective of several conditions such as surgical approach (transperitoneal vs. retroperitoneal) [8]. Such features of the Xi system may allow surgeons, even those without adequate experience with robotic surgery, to acquire the surgical skills required for RAPN.

Here, we wish to emphasize several limitations of this study. First, this was a retrospective comparative study with a small sample size. Second, it is difficult to present the data concerning long-term renal functional as well as prognostic issues due to the short observation period. Finally, RAPN for all patients was performed by a single surgeon, whereas multiple surgeons, including those who had little experience with major surgeries, were involved in OPN. This difference may affect the present outcomes.

In conclusion, we here described our initial experience with RAPN for 50 patients with small renal masses using the da Vinci Xi surgical system, and compared the outcomes of RAPN with those of conventional OPN at our institution. After adjusting backgrounds between the OPN and RAPN groups by propensity-score matching, no significant differences in the major characteristics were noted between these 2 groups; however, RAPN was found to be superior to OPN based on several important perioperative outcomes. Furthermore, trifecta outcome after RAPN was also markedly favorable compared with that after OPN, and the selection of RAPN appeared to be independently associated with improved trifecta outcome. Collectively, these findings suggest that it is possible to perform RAPN with the latest robotic platform, da Vinci Xi, and achieve satisfactory perioperative outcomes after PN for patients with small renal masses. 


\section{References}

$\checkmark 1$ Gill IS, Aron M, Gervais DA, Jewett MA: Clinical practice. Small renal mass. N Engl J Med 2010;362:624-634.

-2 Aboumarzouk OM, Stein RJ, Eyraud R, Eyraud R, Haber GP, Chlosta PL, Somani BK, Kaouk JH: Robotic versus laparoscopic partial nephrectomy: a systematic review and meta-analysis. Eur Urol 2012;62:1023-1033.

-3 Tanagho YS, Figenshau RS, Bhayani SB: Technique, outcomes, and evolving role of extirpative laparoscopic and robotic surgery for renal cell carcinoma. Surg Oncol Clin N Am 2013;22:91-109.

-4 Wu Z, Li M, Liu B, Cai C, Ye H, Lv C, Yang Q, Sheng J, Song S, Qu L, Xiao L, Sun Y, Wang: Robotic versus open partial nephrectomy: a systematic review and meta-analysis. PLoS One 2014;9:e94878.

5 Xia L, Wang X, Xu T, Guzzo TJ: Systematic review and meta-analysis of comparative studies reporting perioperative outcomes of robot-assisted partial nephrectomy versus open partial nephrectomy. J Endourol 2017;31:893-909.

-6 Ficarra V, Minervini A, Antonelli A, Bhayani S, Guazzoni G, Longo N, Martorana G, Morgia G, Mottrie A, Porter J, Simeone C, Vittori G, Zattoni F, Carini M: A multicentre matched-pair analysis comparing robot-assisted versus open partial nephrectomy. BJU Int 2014;113:936-941.

-7 Kim DH, Kim H, Kwak S, Baek K, Na G, Kim JH, Kim SH: The settings, pros and cons of the new surgical robot da Vinci Xi system for transoral robotic surgery (TORS): a comparison with the popular da Vinci Si system. Surg Laparosc Endosc Percutan Tech 2016;26:391-396.
Gettman M, Rivera M: Innovations in robotic surgery. Curr Opin Urol 2016;26:271-276.

-9 Ficarra V, Rossanese M, Gnech M, Novara G, Mottrie A: Outcomes and limitations of laparoscopic and robotic partial nephrectomy. Curr Opin Urol 2014;24:441-447.

10 Furukawa J, Miyake H, Tanaka K, Sugimoto M, Fujisawa M: Console-integrated real-time three-dimensional image overlay navigation for robot-assisted partial nephrectomy with selective arterial clamping: early single-centre experience with 17 cases. Int J Med Robot 2014;10:385-390.

11 Kallingal GJ, Swain S, Darwiche F, Punnen S, Manoharan M, Gonzalgo ML, Parekh DJ: Robotic partial nephrectomy with the da Vinci Xi. Adv Urol 2016;2016:9675095.

$\checkmark 12$ Paulucci DJ, Abaza R, Eun DD, Hemal AK, Badani KK: Robot-assisted partial nephrectomy: continued refinement of outcomes beyond the initial learning curve. BJU Int 2017;119:748-754.

13 Zargar H, Allaf ME, Bhayani S, Stifelman M, Rogers C, Ball MW, Larson J, Marshall S, Kumar R, Kaouk JH: Trifecta and optimal perioperative outcomes of robotic and laparoscopic partial nephrectomy in surgical treatment of small renal masses: a multi-institutional study. BJU Int 2015;116:407-414.

14 Maddox M, Mandava S, Liu J, Boonjindasup A, Boonjindasup A, Lee BR: Robotic partial nephrectomy for clinical stage $\mathrm{T} 1 \mathrm{~b}$ tumors: intermediate oncologic and functional outcomes. Clin Genitourin Cancer 2015;13:9499.
15 Veeratterapillay R, Addla SK, Jelley C, Bailie J, Rix D, Bromage S, Oakley N, Weston R, Soomro NA: Early surgical outcomes and oncological results of robot-assisted partial nephrectomy: a multicentre study. BJU Int 2017;120:550-555.

16 Kim DK, Kim LH, Raheem AA, Shin TY, Alabdulaali I, Yoon YE, Han WK, Rha KH: Comparison of trifecta and pentafecta outcomes between $\mathrm{T} 1 \mathrm{a}$ and $\mathrm{T} 1 \mathrm{~b}$ renal masses following robot-assisted partial nephrectomy (RAPN) with minimum one year follow up: can RAPN for T1b renal masses be feasible? PLoS One 2016;11:e0151738.

17 Porpiglia F, Mari A, Bertolo R, Antonelli A, Bianchi G, Fidanza F, Fiori C, Furlan M, Morgia G, Novara G, Rocco B, Rovereto B, Serni S, Simeone C, Carini M, Minervini A: Partial nephrectomy in clinical T1b renal tumors: multicenter comparative study of open, laparoscopic and robot-assisted approach (the RECORd Project). Urology 2016;89:45-51.

18 Khalifeh A, Autorino R, Hillyer SP, Laydner H, Eyraud R, Panumatrassamee K, Long JA, Kaouk JH: Comparative outcomes and assessment of trifecta in 500 robotic and laparoscopic partial nephrectomy cases: a single surgeon experience. J Urol 2013;189:12361242.

19 Tufek I, Mourmouris P, Doganca T, Obek C, Argun OB, Tuna MB, Keskin MS, Kural AR: Robot-assisted partial nephrectomy for T1b tumors: strict trifecta outcomes. JSLS 2017;21:e2016.00113.

-20 Carneiro A, Sivaraman A, Sanchez-Salas R, Di Trapani E, Barret E, Rozet F, Galiano M, Pizzaro FU, Doizi S, Cathala N, Mombet A, Prapotnich D, Cathelineau X: Evolution from laparoscopic to robotic nephron sparing surgery: a high-volume laparoscopic center experience on achieving 'trifecta' outcomes. World J Urol 2015:33:2039-2044. 